\title{
Azithromycin attenuates acute radiation-induced lung injury in mice
}

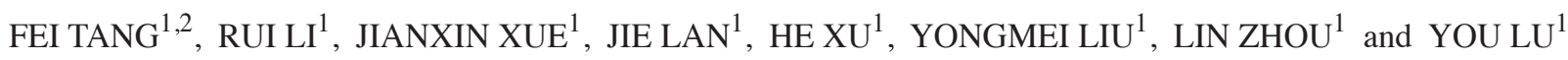 \\ ${ }^{1}$ Department of Thoracic Oncology, Cancer Center and State Key Laboratory of Biotherapy, \\ West China Hospital, Sichuan University, Chengdu, Sichuan 610041; ${ }^{2}$ Department of Medical \\ Oncology, Guizhou Province People's Hospital, Guiyang, Guizhou 550002, P.R. China
}

Received January 1, 2016; Accepted June 9, 2017

DOI: $10.3892 / \mathrm{ol} .2017 .6813$

\begin{abstract}
Radiation-induced lung injury (RILI) is a common and major obstacle in thoracic cancer radiotherapy, resulting in considerable morbidity and limiting the dose of radiation. However, an effective treatment option remains to be established. Therefore, the present study aimed to investigate the effects of azithromycin (AZM) in acute RILI with a mouse model. In the present study, C57BL/6 mice were given a single thoracic irradiation of $16 \mathrm{~Gy}$ and administered orally with AZM. The lung histopathological findings, the levels of malondialdehyde (MDA; an indicator of oxidative damage) and the concentration of pro-inflammatory and pro-fibrotic cytokines in plasma were assessed on 28 day following irradiation. In addition, the total cell counts in bronchoalveolar lavage fluid (BALF), the pro-inflammatory and pro-fibrotic cytokine gene expression in lung tissue were evaluated on day 7, 14 and 28 following irradiation. Administration with AZM markedly alleviated acute RILI as indicated by hematoxylin and eosin and Masson staining. The levels of MDA and total cell counts in BALF significantly reduced in AZM treated mice. AZM also down-regulated the concentration and mRNA expression of interleukin (IL)-1 $\beta$, IL-6, tumor necrosis factor- $\alpha$ and transforming growth factor- $\beta 1$. In addition, AZM attenuated the irradiation-induced increases in the mRNA expression of fibrotic markers $(\alpha$-smooth muscle actin and $\alpha-1$ type I collagen). AZM treatment mitigated the radiation-induced acute lung injury possibly by its anti-inflammatory and anti-fibrotic effects.
\end{abstract}

Correspondence to: Mr. You Lu, Department of Thoracic Oncology, Cancer Center and State Key Laboratory of Biotherapy, West China Hospital, 37 Guoxue Lane, Chengdu, Sichuan 610041, P.R. China

E-mail: radyoulu@hotmail.com

Key words: azithromycin, radiation-induced lung injury, inflammation, fibrosis, cytokine

\section{Introduction}

Radiation therapy (RT) is an essential therapeutic modality for treating thoracic malignancies, including lung cancer and breast cancer (1). Unfortunately, radiation-induced cell death is not confined to tumors. Normal lung tissue is damaged due to the generation of reactive oxygen species and subsequent inflammation and fibrosis (2). Radiation-induced lung injury (RILI) is a common and major obstacle in thoracic cancer radiotherapy, which results in considerable morbidity and limits the dose of radiation (3). The clinical incidence of radiation-induced pneumonitis ranges from 5 to $30 \%$ (4) and is an increasingly prevalent cause of morbidity and mortality (5). Therefore, alleviating RILI is critical for improving tumor control and patient quality of life. However at present, there is no known effective therapeutic strategy to prevent, mitigate, and treat RILI.

The exact pathophysiology of RILI is not completely understood, but the evidence suggests that inflammation has a central role in the initiation and establishment of RILI, especially acute RILI (6). It is generally hypothesized that this process is regulated by the release and activation of various pro-inflammatory and pro-fibrotic cytokines by damaged and activated cells, including interleukin-1 $\beta$ (IL-1 $\beta$ ), interleukin-6 (IL-6), tumor necrosis factor- $\alpha$ (TNF- $\alpha$ ) and transforming growth factor- $\beta 1$ (TGF- $\beta 1)$ (2).

Azithromycin (AZM) is a second-generation macrolide antibiotic with broad-spectrum efficacy against gram-positive, gram-negative and atypical pathogens. In addition to its antibiotic activity, several studies have established that AZM possesses anti-inflammatory and immunomodulatory properties, and reaches very high and stable lung concentrations (7). AZM treatment has been demonstrated to decrease pulmonary exacerbations and improve lung function in patients with cystic fibrosis $(\mathrm{CF})(8,9)$, chronic obstructive pulmonary disease and non-CF bronchiectasis $(10,11)$. AZM has also been shown to be beneficial in lung transplantation for the prevention and treatment of chronic allograft rejection (12). The mechanisms of action involved in the anti-inflammatory and immunomodulatory properties of AZM are being investigated, but remain unclear and are independent of its traditional antimicrobial activity $(13,14)$. AZM was also previously reported to inhibit mRNA and protein expression of pro-inflammatory cytokines 
(Tumor necrosis factor- $\alpha$ and interleukin-1 $\beta$ ) in cultured human corneal epithelial cells stimulated by Toll-like receptor agonists (15).

The therapeutic potential and usefulness of AZM in RILI treatment has not been studied. In the present study, the authors investigated the effects of AZM on acute RILI in a C57BL/6 mouse model.

\section{Materials and methods}

Animals and irradiation. Female C57BL/6 mice $(n=65$; weight, 18-20 g, 8 weeks old) were purchased from the Experimental Animal Center, Chinese Academy of Medical Sciences (Beijing, China) and kept under conventional pathogen-free conditions. The mice were maintained at $23 \pm 2^{\circ} \mathrm{C}$, with a relative humidity of $50 \pm 5 \%$, artificial lighting from 08:00-20:00 and 13-18 air changes/h. The mice were given a standard diet for laboratory rats or mice and water ad libitum. The investigation was performed in compliance with the Guide for the Care and Use of Laboratory Animals published by the US National Institutes of Health (16), and approved by the Animal Care and Use Committee of Sichuan University (Chengdu, China).

The mice were anesthetized and subjected to $16 \mathrm{~Gy}$ whole thoracic irradiation using $6 \mathrm{MV}$ X-rays (Varian Clinac 600C X-ray; Varian Medical Systems, Palo Alto, CA, USA). The head, abdomen, and extremities were shielded with lead strips. Non-irradiated mice were treated in the same manner but without radiation.

Treatment protocol. AZM (Dalian Meilun Biology Technology Co., Ltd, Liaoning, China) was dissolved in vehicle (3.5\% ethyl alcohol absolute and $96.5 \%$ corn oil). The recommended dose in humans is $500 \mathrm{mg}$, which is $\sim 10 \mathrm{mg} / \mathrm{kg}$ in mice. Furthermore, a higher dosage of AZM is required in mice than in humans due to rapid liver metabolism in mice, resulting in an elimination half-life of $2.3 \mathrm{~h}$ compared with $68 \mathrm{~h}$ in humans $(17,18)$. In order to select an appropriate dose, 10 and $100 \mathrm{mg} / \mathrm{kg} /$ day of AZM were used in the pilot study.

In the preliminary study, the mice were randomly divided into four groups: Control (non-irradiated control + vehicle, $\mathrm{n}=5$ ), RT (irradiation + vehicle, $\mathrm{n}=5$ ), AZM-10 (irradiation $+10 \mathrm{mg} / \mathrm{kg} /$ day $\mathrm{AZM}, \mathrm{n}=5$ ) and AZM-100 groups (irradiation $+100 \mathrm{mg} / \mathrm{kg} /$ day $\mathrm{AZM}, \mathrm{n}=5$ ). In the formal study, the mice were randomly divided into three groups: Control (non-irradiated control + vehicle, $\mathrm{n}=15$ ), RT (irradiation + vehicle, $\mathrm{n}=15$ ) and AZM groups (irradiation + AZM, $\mathrm{n}=15)$.

One day prior to irradiation, the mice received AZM $(10 \mathrm{mg} / \mathrm{kg}$ or $100 \mathrm{mg} / \mathrm{kg}$ ) or the same volume of vehicle alone $(5 \mathrm{ml} / \mathrm{kg}$ ) by gavage. Following irradiation, the animals were treated with the original protocol, as described in the preliminary study, once daily until sacrificed under anesthesia.

Liquichip assay. At anesthesia, peripheral blood was collected following enucleation of eyeball, and the blood was permitted to clot at $4^{\circ} \mathrm{C}$ for $24 \mathrm{~h}$ and centrifuged at $1,500 \mathrm{x} \mathrm{g}$ for $15 \mathrm{~min}$. The plasma was collected and stored at $-80^{\circ} \mathrm{C}$ until analysis. The concentration of cytokine in the plasma was assayed using a Mouse Cytokine/Chemokine liquichip kit (EMD Millipore,
Billerica, MA, USA). As TGF- $\beta 1$ was not included in this kit, a separate TGF- $\beta 1$ liquichip kit was used (EMD Millipore).

Bronchoalveolar lavage fluid (BALF) analysis. On day 7, 14 and 28 following irradiation, the mice were sacrificed and the thorax was dissected. The lung tissues were exposed, and the right lobe of lung was ligated with a 6-0 suture, while the left lung was not. An open tracheotomy was performed, and a small plastic tube was inserted into the trachea. To obtain BALF, ice-cold PBS $(0.35 \mathrm{ml})$ was infused into the lung and withdrawn via tracheal cannulation three times (total volume, $1.05 \mathrm{ml})$. BALF was centrifuged $\left(400 \mathrm{x} \mathrm{g}, 15 \mathrm{~min}, 4^{\circ} \mathrm{C}\right)$, and the cell pellet was suspended in $1 \mathrm{ml}$ modified Hank's balanced salt solution. The total number of nucleated cells was counted under a light microscope (Imager A2; Zeiss AG, Oberkochen, Germany). Differential cell count in BALF was performed in a double-blind manner by two independent observers.

Histopathology. The lung tissues were fixed with $10 \%$ formalin and embedded in paraffin. The tissue sections (thickness, $4 \mu \mathrm{m}$ ) were stained with Mayer's hematoxylin (H) for $30 \mathrm{sec}$ and eosin (E) for $20 \mathrm{sec}$ at room temperature and Masson's trichrome (ponceau red acid magenta dye for $10 \mathrm{~min}$ and aniline blue for 5-10 min) at room temperature. Images of the slides were obtained using a digital camera mounted on a light microscope (Imager A2; Zeiss AG). Each H\&E tissue section was given a score between $0-4$ based on the area affected by interstitial inflammation, alveolar wall thickening, peribronchial inflammation and interstitial edema as follows: Score $0, \leq 10 \% ; 1$, $\leq 30 \% ; 2 \leq 50 \% ; 3, \leq 70 \%$ and $4, \geq 70 \%$. A mean inflammation score was determined for each group of mice (19). The grade of fibrosis of each section stained with Masson's trichrome was evaluated with a modified scale of $0-8$, as previously reported (20). Briefly, on a scale of $0-8$, grade 0 represents normal lung and grade 8 represents total fibrous obliteration of the field. This evaluation was performed by two blind independent observers (Department of Thoracic Oncology, Cancer Center and State Key Laboratory of Biotherapy, West China Hospital, Sichuan University, Chengdu, China).

Malondialdehyde (MDA) activity assay. The concentration of MDA was determined in plasma and lysates of radiated lung tissue by using the MDA assay kit (Nanjing Jiancheng Bio-engineering Institute, Jiangsu, China), according to the manufacturer's instructions. The MDA levels were expressed as $\mathrm{nmol} / \mathrm{ml}$ for plasma samples and $\mathrm{nmol} / \mathrm{mg}$ of tissue for lung tissue homogenate.

Quantitative reverse transcription polymerase chain reaction (RT-qPCR). A total of $100 \mathrm{mg}$ irradiated lung tissue was freshly isolated from each sample. Total RNA was isolated with TRIzol reagent (Invitrogen; Thermo Fisher Scientific, Inc., Waltham, MA, USA), and $1 \mathrm{mg}$ total RNA from each sample was used for first-strand complementary DNA synthesis $\left(37^{\circ} \mathrm{C}\right.$ for $15 \mathrm{~min} ; 85^{\circ} \mathrm{C}$ for $\left.5 \mathrm{sec}\right)$ with a RT-PCR kit (Takara Bio, Inc., Otsu, Japan). RT-qPCR $\left(95^{\circ} \mathrm{C}\right.$ for $1 \mathrm{~min} ; 95^{\circ} \mathrm{C}$ for $10 \mathrm{sec} ; 58^{\circ} \mathrm{C}$ for $10 \mathrm{sec} ; 72^{\circ} \mathrm{C}$ for $10 \mathrm{sec}$; all for 40 cycles) was performed with the SYBR RT-PCR kit (Takara Bio, Inc.) on the Chromo4 Real-time PCR system (Bio-Rad Laboratories, Inc., Hercules, CA, USA). The level 
Table I. Primer sequences for quantitative reverse transcription polymerase chain reaction.

\begin{tabular}{lll}
\hline Gene & \multicolumn{1}{c}{ Forward (5'-3') } & \multicolumn{1}{c}{ Reverse (5'-3') } \\
\hline IL-1 $\beta$ & TTCTTGGGACTGATGCTG & CTCATTTCCACGATTTCCC \\
IL-6 & CAGGCTCCGAGATGAACAA & CAGACTCCACTTTGCTCTTGAC \\
TNF- $\alpha$ & CTGTGAAGGGAATGGGTGTT & CAGGGAAGAATCTGGAAAGGTC \\
TGF- $\beta 1$ & ATGGTGGACCGCAACAAC & AGCCACTCAGGCGTATCAG \\
$\alpha-S M A$ & TGCTGGACTCTGGAGATGGT & ATCTCACGCTCGGCAGTAGT \\
COL1A 1 & ACGCCATCAAGGTCTACTGC & CGGGAATCCATCGGTCAT \\
GAPDH & GGTGAAGGTCGGTGTGAACG & CTCGCTCCTGGAAGATGGTG
\end{tabular}

COL1A1, $\alpha$-1 type I collagen; TGF, transforming growth factor; IL, interleukin; $\alpha$-SMA, smooth muscle actin.

of GAPDH mRNA in each sample was used as an internal control. All reactions were performed in duplicate, and the results were analyzed by the $2^{-\Delta \Delta \mathrm{Cq}}$ method (21). The primer sequences are stated in Table I.

Western blot analysis. The lung tissues were homogenized in ice-cold RIPA lysis buffer with protease and phosphatase inhibitors (Nanjing KeyGen Biotech Co., Ltd., Nanjing, China). Homogenates containing $30 \mu \mathrm{g}$ tissue lysate were separated by $10 \%$ SDS-PAGE and transferred to polyvinylidene difluoride membranes (EMD Millipore). The buffer used for blocking was 5\% skimmed milk for $1 \mathrm{~h}$ at room temperature. The membranes were incubated with rabbit monoclonal TGF- $\beta 1$ antibodies (1:500; sc-146; Santa Cruz Biotechnology, Inc., Dallas, TX, USA) and antibodies against $\beta$-actin, which were used as a loading control (1:1,000; cat. no. sc-47778; Santa Cruz Biotechnology, Inc., Dallas, TX, USA), overnight at $4^{\circ} \mathrm{C}$. Immunoreactivity was detected using horseradish peroxidase-conjugated mouse anti-rabbit immunoglobulin G antibody (1:5,000; cat. no. sc-2357; Santa Cruz Biotechnology, Inc.) in blocking solution for $1 \mathrm{~h}$ at room temperature. Immunoreactivity was detected using an enhanced chemiluminescence kit (EMD Millipore). The western blots were imaged and analyzed by The ChemiDoc MP Imaging System of BIO-RAD and the software used was Image Lab 5.2.1 (Bio-Rad Laboratories, Inc.).

Statistical analysis. Data are presented as the mean \pm standard error. The data from different groups during various time points were compared using one-way analysis of variance. $\mathrm{P}<0.05$ was considered to indicate a statistically significant difference. Statistical analyses were carried out using GraphPad Prism 6.0 (GraphPad Software, Inc., La Jolla, CA, USA).

\section{Results}

AZM treatment attenuates RILI histopathology. The experimental protocol is shown in Fig. 1A. In the preliminary study, the authors evaluated RILI-associated histological changes on day 28 using H\&E and Masson stained lung sections. Compared with the control group, lung tissue in the RT group showed markedly thickened alveolar walls, collapsed alveoli and marked inflammatory pathological changes, including local inflammatory cell infiltration and inflammatory exudation (Fig. 1B). By contrast, treatment with AZM decreased the thickness of alveolar walls and alleviated interstitial edema (Fig. 1B). Masson staining showed radiation-induced collagen deposition in parts of the lung tissues, and AZM treatment attenuated this deposition (Fig. 1C). Similarly, when lung tissue inflammation and grade of fibrosis were evaluated, the increased inflammation score and grade of fibrosis caused by irradiation were significantly decreased following $100 \mathrm{mg} / \mathrm{kg} /$ day AZM treatment (both $\mathrm{P}<0.01$; Fig. 1D and E). Unfortunately, compared with the RT group, the score and grade in the AZM-10 group were lower. However, the differences in score and grade in the AZM-10 group were not statistically significant (both $\mathrm{P}>0.05$; Fig. 1D and E).

AZM treatment reduces the level of lipid peroxidation. The present authors measured the levels of MDA in plasma and lung tissue homogenates in order to investigate the effects of AZM on radiation-induced lipid peroxidation. Irradiation treatment increased the levels of MDA. However, the levels of MDA in the AZM-100 group significantly decreased in plasma and lung tissue (both $\mathrm{P}<0.01$ vs. RT group; Fig. 2A and $\mathrm{B}$ ). These results indicated that $100 \mathrm{mg} / \mathrm{kg} /$ day AZM was effective in reducing the level of lipid peroxidation. Compared with the RT group, the levels of MDA in the AZM-10 group were lower in the plasma and lung tissue. However, no significant differences were observed in plasma and lung tissue. Therefore, $100 \mathrm{mg} / \mathrm{kg} /$ day was selected as the high AZM dose in subsequent experiments.

AZM administration decreases total cell counts in BALF. In the formal study, the mice were sacrificed under anesthesia on day 7, 14 and 28 post-irradiation. The authors evaluated the effect of AZM on total cell counts in BALF following irradiation (Fig. 3). In the RT group, the counts decreased on day 7 compared with the control, and the levels in the RT group decreased further on day 14 (day 7 and 14 vs. control, $\mathrm{P}<0.01$; Fig. 3). By day 28, a marked increase was observed compared with the control (day 28 vs. control). However, in the AZM-100 group, total cell counts were not significantly different to those in the control group on day 7 ( $P>0.05$ vs. control. The total cell counts in the AZM-100 group were increased on day 14 compared with the RT group $(\mathrm{P}<0.05$ vs. RT group; Fig. 3), while the influx of total cells 

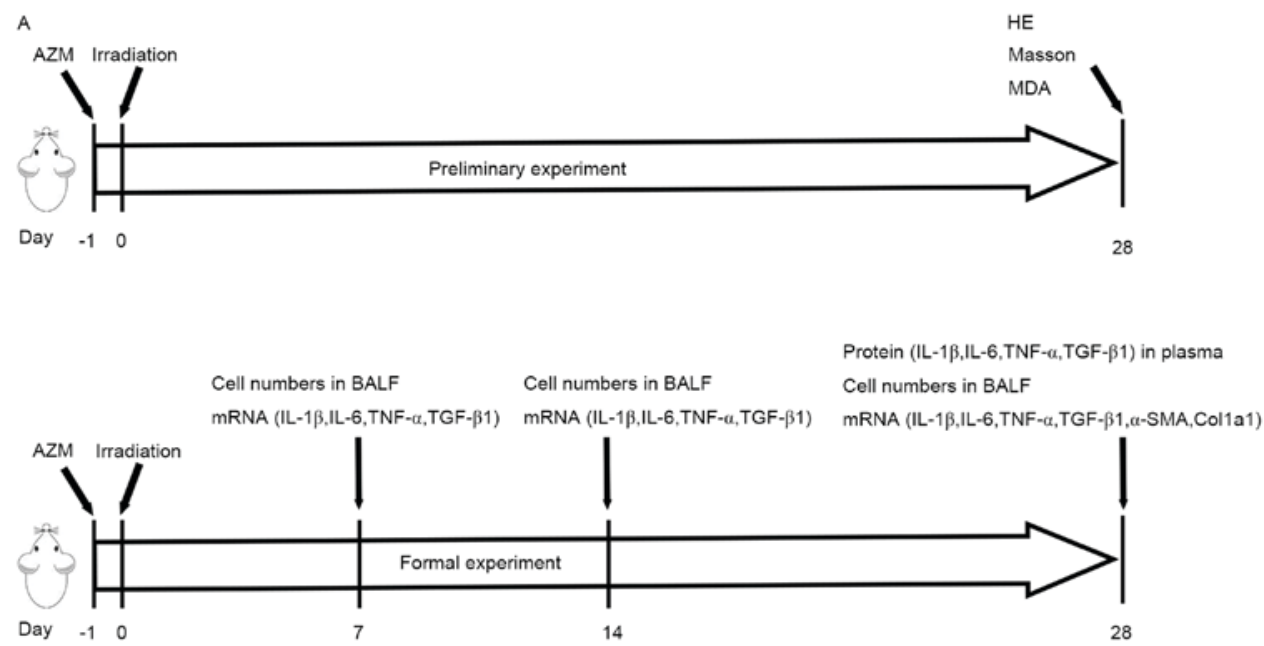

B

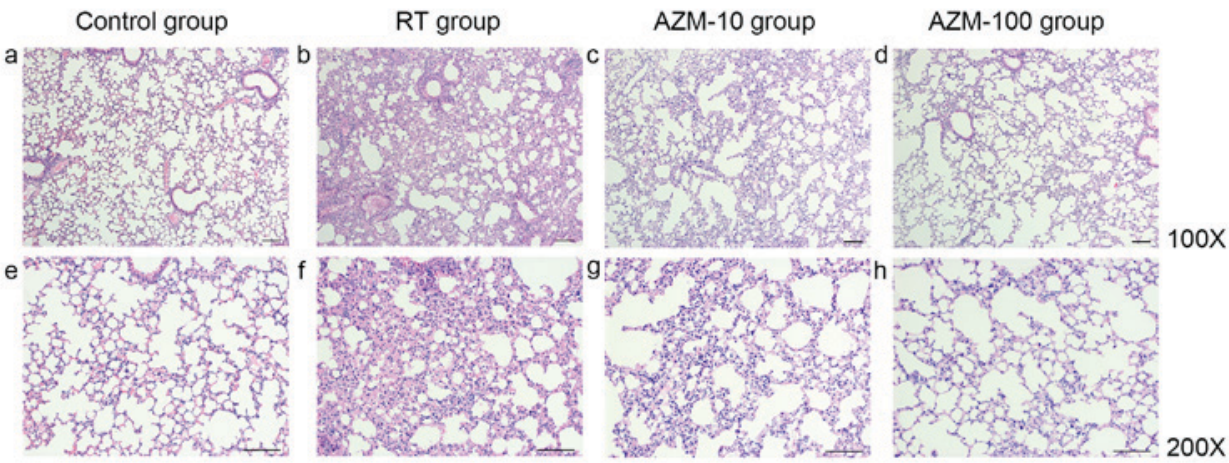

C

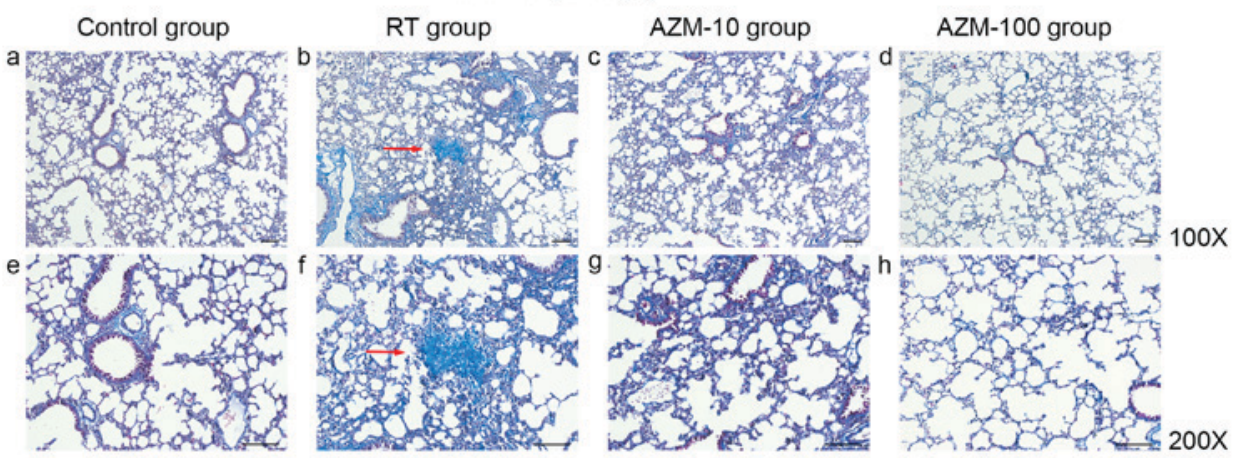

Figure 1. Schematic diagram of the experimental protocol and the effect of AZM on histological changes in the control, RT, AZM-10 and AZM-100 groups on day 28 following irradiation. (A) The mouse thorax was irradiated with $16 \mathrm{~Gy} \mathrm{X}$-ray. One day prior to radiation, the mice received AZM or vehicle alone and were administered with the original protocol, as described in the preliminary study, once daily until the mice were sacrificed under anesthesia. Lung tissues, plasma and bronchoalveolar lavage fluid were collected at indicated time points for each experiment. (B) Representative photomicrographs of hematoxylin and eosin stained lung sections. Characteristic morphology of each group is shown at magnifications (a-d) 100x and (e-h) 200x. Scale bar, $100 \mu \mathrm{m}$. (C) Representative photomicrographs of Masson stained lung sections. Characteristic morphology of each group is shown at (a-d) 100x and (e-h) 200x. Examples of collagen deposition lesions (red arrows) are marked. Scale bar, $100 \mu \mathrm{m}$. Control, non-irradiated control + vehicle; RT group, irradiation + vehicle; AZM-10 group, irradiation $+10 \mathrm{mg} / \mathrm{kg} / \mathrm{day}$ AZM; AZM-100 group; irradiation + $100 \mathrm{mg} / \mathrm{kg} / \mathrm{day}$ AZM. AZM, azithromycin; BALF, bronchoalveolar lavage fluid; COL1A1, $\alpha$-1 type I collagen; H\&E, hematoxylin and eosin; MDA, malondialdehyde; TNF, tumor necrosis factor; TGF, transforming growth factor; IL, interleukin; $\alpha$-SMA, smooth muscle actin.

in the AZM-100 group was significantly decreased on day 28 compared with the RT group (P<0.05; Fig. 3).

AZM treatment reduces the levels of pro-inflammatory cytokine expression in plasma. The concentrations of pro-inflammatory cytokines in plasma, including IL-1 $\beta$, IL-6 and TNF- $\alpha$, were measured by liquichip on day 28 following irradiation. Thorax irradiation resulted in the abundant production of IL-1 $\beta$, IL- 6 and TNF- $\alpha$, and the plasma levels of these cytokines significantly increased in RILI mice on day 28 (all $\mathrm{P}<0.05$ vs. control group). By contrast, AZM treatment significantly decreased the irradiation-induced protein 

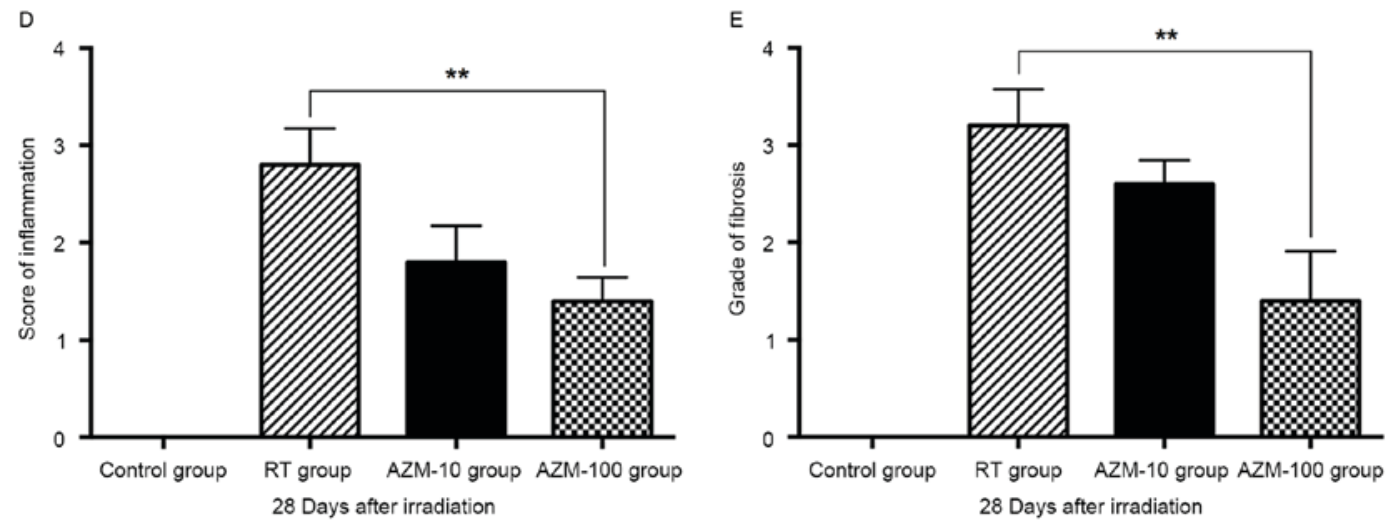

Figure 1. Continued. (D) Scoring of lung tissue inflammation as assessed by one-way ANOVA. Data are represented as the mean \pm standard error. ${ }^{* *} \mathrm{P}<0.01$, RT group vs. AZM-100 group; $\mathrm{n}=5$ per group. (E) Grading of lung tissue fibrosis as assessed by one-way ANOVA. Data are represented as the mean \pm standard error. ${ }^{* *} \mathrm{P}<0.01$, RT group vs. AZM-100 group; $\mathrm{n}=5$ per group. Control, non-irradiated control + vehicle; RT group, irradiation + vehicle; AZM-10 group, irradiation $+10 \mathrm{mg} / \mathrm{kg} /$ day AZM; AZM-100 group; irradiation $+100 \mathrm{mg} / \mathrm{kg} /$ day AZM. ANOVA, one-way analysis of variance; AZM, azithromycin.
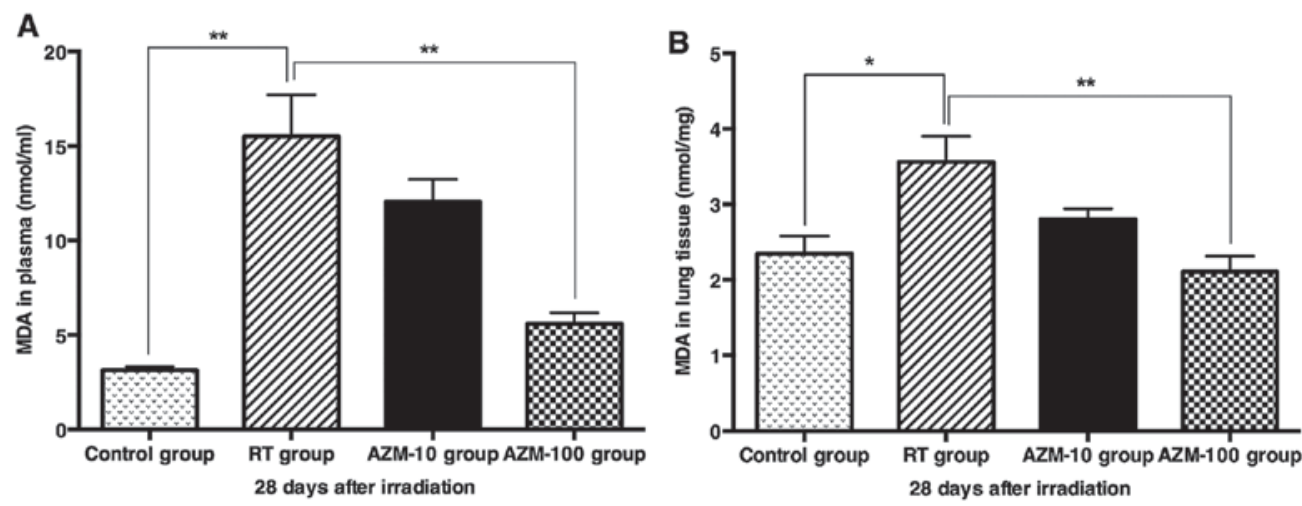

Figure 2. Effect of AZM on the levels of MDA. Changes in (A) plasma and (B) lung tissues in the control group, RT, AZM-10 and AZM-100 groups on 28 day following irradiation. Data are represented as the mean \pm standard error. In (A) both Control vs. RT group and RT group vs. AZM-100 group: * $\mathrm{P}<0.01$. In (B) control vs. RT group: " $\mathrm{P}<0.05$, RT group vs. AZM-100 group: *" $\mathrm{P}<0.01 ; 5$ samples/group. Control, non-irradiated control + vehicle; RT group, irradiation + vehicle; AZM-10 group, irradiation + 10 mg $/ \mathrm{kg} /$ day AZM; AZM-100 group; irradiation + $100 \mathrm{mg} / \mathrm{kg} / \mathrm{day}$ AZM. AZM, azithromycin; MDA, malondialdehyde.

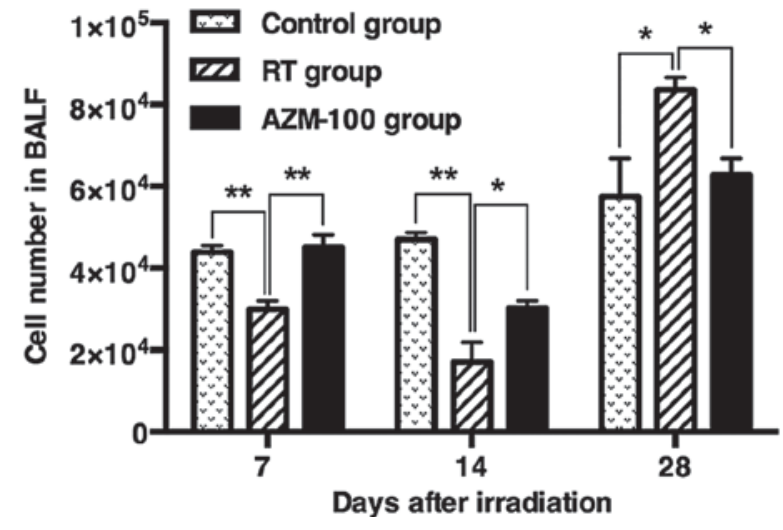

Figure 3. Effect of AZM on changes in total cell counts in BALF on day 7, 14 and 28 following irradiation. Control group vs. RT group and AZM-100 group at the same time points, by one-way analysis of variance. Data are represented as the mean \pm standard error. Day 7: Control vs. RT group and RT group vs. AZM-100 group, ${ }^{* *} \mathrm{P}<0.01$; day 14: Control vs. RT group, ${ }^{* *} \mathrm{P}<0.01$ and $\mathrm{RT}$ group vs. AZM-100 group, "P<0.05; day 28: Control vs. RT group and RT group vs. AZM-100 group, ${ }^{*} \mathrm{P}<0.05 ; 5$ samples/group. Control, non-irradiated control + vehicle; RT group, irradiation + vehicle; AZM-10 group, irradiation $+10 \mathrm{mg} / \mathrm{kg} /$ day AZM; AZM-100 group; irradiation + $100 \mathrm{mg} / \mathrm{kg} /$ day AZM. AZM, azithromycin; BALF, bronchoalveolar lavage fluid. release of IL-1 $\beta$, IL- 6 and TNF- $\alpha$ in plasma compared with the RT group (Fig. 4A-C).

AZM treatment reduces pro-inflammatory cytokine gene expression in lung tissue. To gain further insight into the effect of AZM on RILI, lung mRNA samples from these mice were measured on day 7, 14 and 28 following irradiation using RT-qPCR. As shown in Fig. 5A and B, irradiation resulted in a slight increase in the levels of IL-1 $\beta$ and IL-6 on day 7, 14 and 28 compared with the control group (Fig. 5A and B). By contrast, AZM treatment significantly inhibited this increase on day 28 (Fig. 5A and B). On day 14 following irradiation, there was an increase in the levels of TNF- $\alpha$ compared with the control group, and this increase was significantly reduced in AZM-treated mice (Fig. 5C).

AZM treatment reduces pro-fibrotic factor expression. The present authors also examined TGF- $\beta 1$ expression in plasma and lung tissue using liquichip or western blotting on day 28 following irradiation. As expected, AZM treatment significantly decreased irradiation-induced TGF- $\beta 1$ 

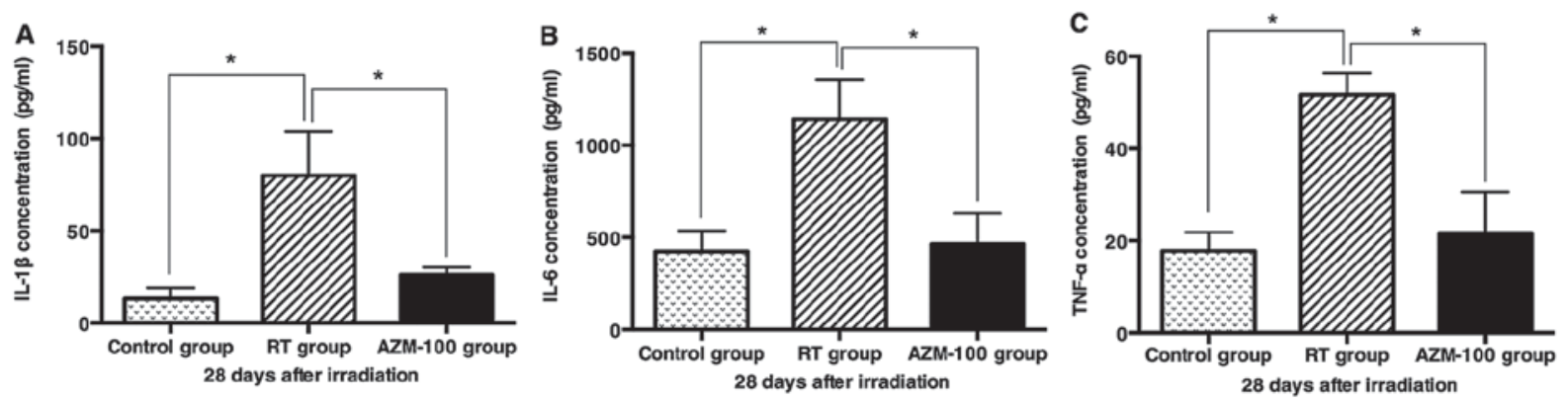

Figure 4. Analysis of concentration of pro-inflammatory cytokines in plasma by Liquichip on 28 day following irradiation. Analysis of (A) IL-1 $\beta$, (B) IL-6 and (C) TNF- $\alpha$ concentration. Data are represented as the mean \pm standard error. Control vs. RT group and RT group vs. AZM-100 group, "P $<0.05 ; 5$ samples/group. Control, non-irradiated control + vehicle; RT group, irradiation + vehicle; AZM-10 group, irradiation + 10 mg/kg/day AZM; AZM-100 group; irradiation $+100 \mathrm{mg} / \mathrm{kg} /$ day AZM. AZM, azithromycin; TNF, tumor necrosis factor; IL, interleukin.
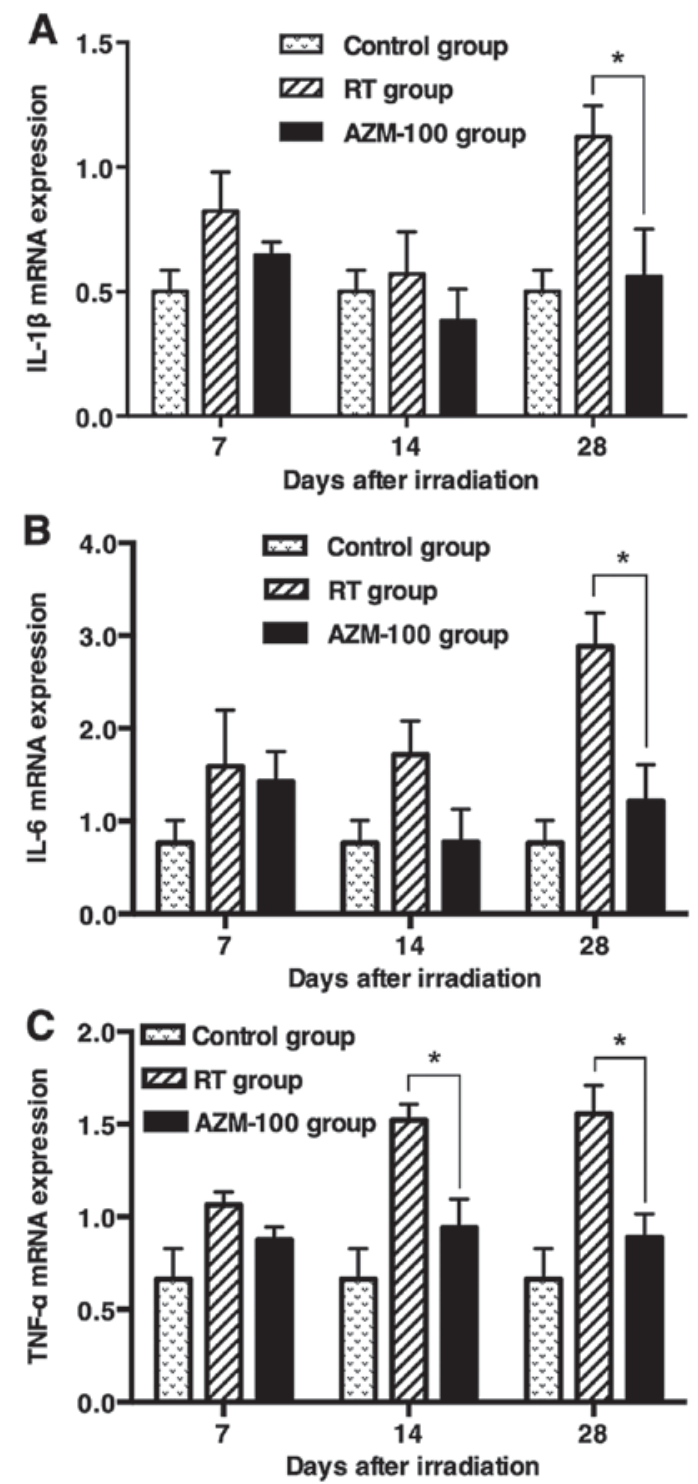

Figure 5. Analysis of changes in pro-inflammatory cytokine gene expression in lung tissues by quantitative reverse transcription polymerase chain reaction on day 7,14 and 28 following irradiation. (A) IL-1 $\beta$ expression. (B) IL-6 expression. (C) TNF- $\alpha$ expression. Data are represented as the mean \pm standard error. In (C) on day 14 , RT group vs. AZM-100 group, ${ }^{*} \mathrm{P}<0.05$; in (A-C) on day 28 , RT group vs. AZM-100 group, ${ }^{*} \mathrm{P}<0.05 ; 5$ samples/group. Control, non-irradiated control + vehicle; RT group, irradiation + vehicle AZM-10 group, irradiation + $10 \mathrm{mg} / \mathrm{kg} /$ day AZM; AZM-100 group; irradiation $+100 \mathrm{mg} / \mathrm{kg} /$ day AZM. TNF, tumor necrosis factor; IL, interleukin. expression in plasma and lung tissues compared with the RT group (Fig. 6A-C). In order to further determine the changes in TGF- $\beta 1$, the authors measured TGF- $\beta 1$ mRNA expression in injured lungs using RT-qPCR on day 7, 14 and 28 following irradiation. The irradiation-induced increase in TGF- $\beta 1$ mRNA expression was significantly decreased in the AZM-100 group on day 7, 14 and 28 (Fig. 6D).

The mRNA expression of $\alpha$-smooth muscle actin ( $\alpha$-SMA) and $\alpha-1$ type 1 collagen (COL1A1) was examined in lung tissue on day 28 following irradiation. The irradiation-induced increase in $\alpha$-SMA and COL1A1 mRNA expression in injured lung tissue significantly decreased in the AZM-100 group (Fig. 6E and F).

\section{Discussion}

In the present study, a murine model was used to investigate the effect of AZM, as a new biological strategy, to ameliorate acute RILI. The results showed that AZM decreased radiation-induced early lung injury. Oxidative stress, inflammatory cell infiltration, cytokine production, and associated gene expression have pivotal roles in the pathogenesis of RILI (22). Although, the exact mechanisms by which RILI is mitigated by AZM are not well described, anti-inflammatory and anti-fibrotic effects may be involved.

Lipid peroxidation is one of the oxidative conversions of polyunsaturated fatty acids to products such as MDA, which is an important indicator of oxidative damage $(23,24)$. In the present study, marked increases in MDA content were observed between week 1 and 24 following whole-lung irradiation, which demonstrated oxidative stress due to radiation-induced pneumonitis and lung fibrosis. Oxidative stress has been previously shown to ameliorate RILI (25). A previous study indicated that AZM decreased the levels of MDA in a pig model of otitis media (26). These findings were confirmed by the results of the present study. In addition, radiation is known to induce the expression of $\mathrm{NO}$ synthase (NOS) and results in nitrotyrosine formation in the lungs (27). Evidence to support the harmful role of NOS in RILI includes a study in which partial attenuation of RILI was observed following treatment with L-nitro-arginine methyl ester, a relatively nonspecific NOS inhibitor (28). Notably, AZM also significantly reduced NOS activity in a rat model of colonic damage (29). 

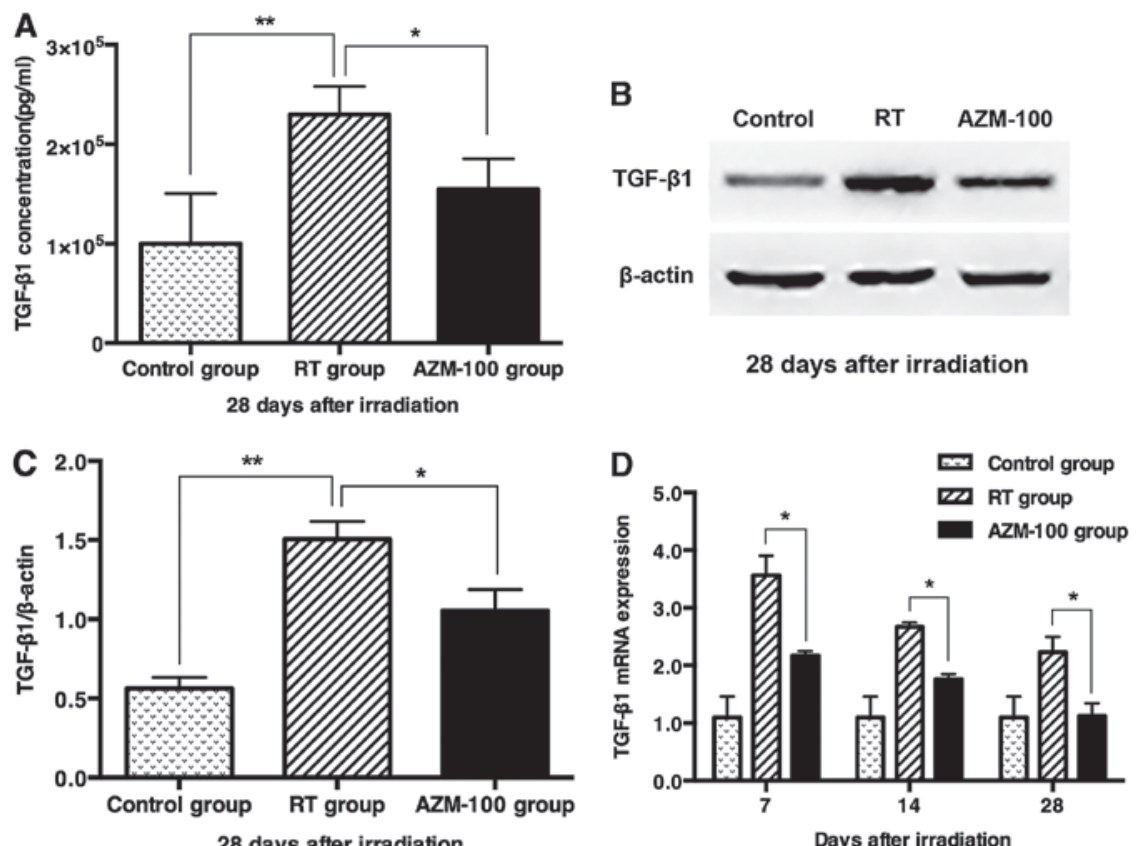

28 days after irradiation

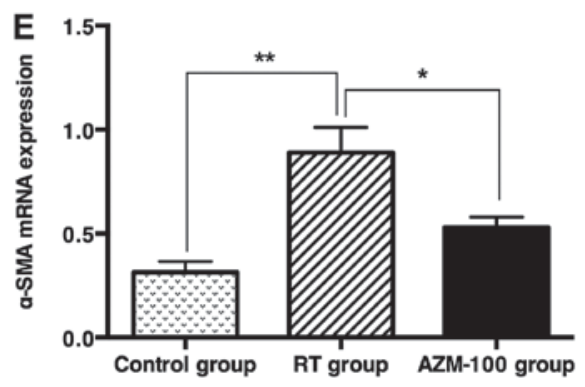

28 days after irradiation

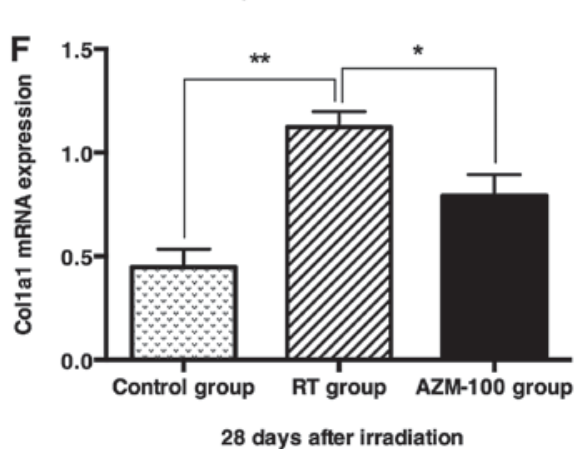

Figure 6. Effect of AZM on changes in the level of pro-fibrotic factor in plasma and lung tissue following irradiation. (A) Changes in TGF- $\beta 1$ concentration in plasma on day 28 following irradiation. (B) Western blot analysis of TGF- $\beta 1$ in lung tissue on day 28 following irradiation. (C) Relative TGF- $\beta 1$ protein expression in lung tissue. Densitometry values were normalized to $\beta$-actin. (D) Changes in TGF- $\beta 1 \mathrm{mRNA}$ expression in lung tissue on day 7,14 and 28 following irradiation. (E) $\alpha$-SMA mRNA expression in lung tissue on 28 day following irradiation. (F) COL1A1 mRNA expression in lung tissue on 28 day following irradiation. Data are represented as the mean \pm standard error. Control vs. RT group, ${ }^{* * *} \mathrm{P}<0.01$; RT group vs. AZM-100 group, "P<0.05; 5 samples/group. Control, non-irradiated control + vehicle; RT group, irradiation + vehicle; AZM-10 group, irradiation + $10 \mathrm{mg} / \mathrm{kg} / \mathrm{day} \mathrm{AZM;} \mathrm{AZM-100} \mathrm{group;}$ irradiation $+100 \mathrm{mg} / \mathrm{kg} /$ day AZM. AZM, azithromycin; COL1A1, $\alpha-1$ type I collagen; TGF, transforming growth factor; IL, interleukin; $\alpha$-SMA, $\alpha$-smooth muscle actin.

Evidence suggests a central role for the inflammatory response in the initiation and establishment of RILI. Local recruitment of inflammatory cells and the production of inflammatory cytokines exert an important role in mediating, amplifying and maintaining the RILI process. Intensive anti-inflammatory treatment mitigates the signs and symptoms of RILI $(30,31)$.

Many studies have investigated radiation-induced lung damage using BALF, as it is thought to reflect the lung inflammatory response $(32,33)$. Previous studies have indicated that most patients did not show clinical symptoms of radiation pneumonitis, and lymphocytosis was not very pronounced. However in symptomatic patients, changes in BALF, including total cellularity and lymphocytosis [which appeared to be mainly activated cluster of differentiation (CD) $4^{+}$cells], were significantly greater compared with asymptomatic patients (34). In the present study, the total number of cells obtained by BALF decreased on day 7 following irradiation and was very low on day 14 . However on day 28 , a marked increase was observed. These dynamic changes are in agreement with previous studies $(35,36)$. A previous study indicated that the late increase in BALF cell number was associated with the development of radiation-induced pulmonary lethality $(35,36)$. As shown in the present study, administration of AZM inhibited these dynamic changes. In particular, the number of cells on day 28 was reduced in the AZM-treated group compared with the control. These results are supported by previous data which showed that AZM treatment decreased total cell counts in BALF in a mouse model of ventilator-associated pneumonia and bleomycin-induced acute lung injury $(37,38)$. In addition, inflammatory cell infiltration or exudation into lung parenchyma appears to have to an important role in the development of RILI. Agents that decrease inflammatory cell exudation have the potential to alleviate RILI (39). The present study demonstrated that AZM markedly reduced inflammatory infiltration in the alveolar septa, therefore alleviating the extent of RILI (as determined by H\&E staining). 
Many studies have indicated that AZM reduces the inflammatory response by decreasing the levels of pro-inflammatory cytokine in lipopolysaccharide- and bleomycin-induced acute lung injury models. These findings are consistent with those in the present study $(40,41)$. Alveolar macrophages have an important role in alveolar physiology. Activated macrophages are the main source of pro-inflammatory cytokines in the early stages of radiation-induced lung disease (42). A number of studies have confirmed that the anti-inflammatory properties of AZM can be attributed, at least partly, to its action on macrophages $(41,43)$. For example, AZM prevented the production of pro-inflammatory cytokines by macrophages in the lipopolysaccharide-induced lung neutrophilia mouse model and inhibited inflammatory cytokine production by J774A.1 macrophage cell lines $(41,43)$. Therefore, the authors hypothesize that AZM regulates inflammatory cytokine production by targeting macrophages following irradiation.

TGF- $\beta$, which is a potent stimulator of collagen protein synthesis, exerts a critical role in the pathogenesis of radiation-induced lung fibrosis (44-46). Gene expression of TGF- $\beta$ has been demonstrated to increase markedly 1-14 days following irradiation, in parallel with changes in fibroblast gene expression of collagen I and fibronectin. The administration of anti-TGF- $\beta 1$ antibody 1 D11 or the TGF- $\beta$ receptor inhibitor LY210976 is now an option for ameliorating RILI (46-48). In a rat model of bleomycin-induced pulmonary fibrosis, the levels of TGF- $\beta$ protein and mRNA were reduced following treatment with AZM in the early stage of pulmonary fibrosis (49). In the present study, it was observed that AZM treatment significantly decreased expression of TGF- $\beta 1, \alpha$-SMA and COL1A1, and grade of fibrosis in Masson stained lung sections, indicating that AZM may contribute to the anti-fibrotic effects of post-irradiation.

Thorax irradiation not only affects macrophages in lung tissue, but also triggers the recruitment of various immune cells into the lung, including $\mathrm{CD} 4^{+} \mathrm{T}$-lymphocytes, which exert a critical role in the pathogenesis of radiation-induced pneumonitis preceding lung fibrosis (50). A pronounced increase in $\mathrm{CD}^{+}$lymphocytes was demonstrated 4 weeks following irradiation. Co-culture of isolated $\mathrm{CD}^{+} \mathrm{T}$ cells from irradiated lungs with fibroblasts resulted in increased collagen production (51). Furthermore, depletion of $\mathrm{CD}^{+}$ $\mathrm{T}$ cells by specific antibodies prior to partial lung irradiation decreased the degree of radiation-induced lung fibrosis (50). As mentioned previously, AZM has also been shown to be beneficial in lung transplantation for the prevention and treatment of chronic allograft rejection due to its immunomodulatory properties (52). AZM treatment significantly decreased CD4 ${ }^{+}$ $\mathrm{T}$ cells in BALF and lung collagen deposition in a murine model of noninfectious lung injury (53). The authors of the present study hypothesize that the anti-fibrotic effects of AZM may be attributed to its immunomodulatory properties.

The authors observed an improvement in radiation-induced lung tissue morphology with high-dose $(100 \mathrm{mg} / \mathrm{kg} / \mathrm{day})$ and low-dose $(10 \mathrm{mg} / \mathrm{kg} /$ day $)$ AZM in the preliminary study. As shown in Fig. 1D and E, compared with the RT group, the inflammation score and the grade of fibrosis in the AZM-10 group were lower, but not significantly different. In addition, a lower but not a statistically significant difference in MDA content was observed in plasma and lung tissue in the AZM-10 group compared with the RT group. These results indicate that $100 \mathrm{mg} / \mathrm{kg} / \mathrm{day}$ is a more appropriate dose for AZM in acute RILI. Future studies are required to determine the optimal dose of AZM with the highest efficacy and minimal dose-limiting toxicities in acute RILI.

RILI refers to a continuous process, which is triggered following lung RT (3). Additionally, acute pneumonitis is associated with radiation fibrosis (39). A limitation in the present study is the lack of a long-term investigation on the survival rate of RILI mice treated with AZM. A future study by the present authors will focus on whether AZM has a similar capacity to ameliorate late RILI and improve survival in mice.

In conclusion, AZM has therapeutic potential in RILI management. The present study demonstrated the beneficial role of AZM in treating RILI, including its anti-inflammatory and anti-fibrotic effects.

\section{Acknowledgements}

The present study was supported by the National Natural Science Foundation of China (grant nos. 81472196 and 81472808). The abstract was presented at the 58th Annual Meeting of the American Society for Radiation Oncology on 25-28 September 2016, Boston, USA and published as abstract no. 3439 in the Proceedings of the American Society for Radiation Oncology Vol. 92 (2). The authors would like to thank the members of the Laboratory of Stem Cell Biology of Sichuan University (Chengdu, China) for their assistance in the present study, and the members of the Department of Radiation Oncology of West China Hospital (Chengdu, China) for their assistance with the dosimetry verification and mouse radiation therapy.

\section{References}

1. Baskar R, Lee KA, Yeo R and Yeoh KW: Cancer and radiation therapy: Current advances and future directions. Int J Med Sci 9: 193-199, 2012.

2. Graves PR, Siddiqui F, Anscher MS and Movsas B: Radiation pulmonary toxicity: From mechanisms to management. Semin Radiat Oncol 20: 201-207, 2010.

3. Sprung CN, Forrester HB, Siva S and Martin OA: Immunological markers that predict radiation toxicity. Cancer Lett 368: 191-197, 2015.

4. Marks LB, Bentzen SM, Deasy JO, Kong FM, Bradley JD, Vogelius IS, El Naqa I, Hubbs JL, Lebesque JV, Timmerman RD, et al: Radiation dose-volume effects in the lung. Int J Radiat Oncol Biol Phys 76 (3 Suppl): S 70-S76, 2010

5. Yamashita H, Nakagawa K, Nakamura N, Koyanagi H, Tago M, Igaki H, Shiraishi K, Sasano N and Ohtomo K: Exceptionally high incidence of symptomatic grade 2-5 radiation pneumonitis after stereotactic radiation therapy for lung tumors. Radiat Oncol 2: 21, 2007.

6. Kong FM and Wang S: Nondosimetric risk factors for radiation-induced lung toxicity. Semin Radiat Oncol 25: 100-109, 2015.

7. Togami K, Chono S and Morimoto K: Distribution characteristics of clarithromycin and azithromycin, macrolide antimicrobial agents used for treatment of respiratory infections, in lung epithelial lining fluid and alveolar macrophages. Biopharm Drug Dispos 32: 389-397, 2011.

8. Spagnolo P, Fabbri LM and Bush A: Long-term macrolide treatment for chronic respiratory disease. Eur Respir J 42: 239-251, 2013.

9. Southern KW, Barker PM, Solis-Moya A and Patel L: Macrolide antibiotics for cystic fibrosis. Cochrane Database Syst Rev 11: CD002203, 2012. 
10. Wong C, Jayaram L, Karalus N, Eaton T, Tong C, Hockey H, Milne D, Fergusson W, Tuffery C, Sexton P, et al: Azithromycin for prevention of exacerbations in non-cystic fibrosis bronchiectasis (EMBRACE): A randomised, double-blind, placebo-controlled trial. Lancet 380: 660-667, 2012.

11. Albert RK, Connett J, Bailey WC, Casaburi R, Cooper JA Jr, Criner GJ, Curtis JL, Dransfield MT, Han MK, Lazarus SC, et al: Azithromycin for prevention of exacerbations of COPD. N Engl J Med 365: 689-698, 2011.

12. Vos R, Vanaudenaerde BM, Verleden SE, De Vleeschauwer SI, Willems-Widyastuti A, Van Raemdonck DE, Schoonis A Nawrot TS, Dupont LJ and Verleden GM: A randomised controlled trial of azithromycin to prevent chronic rejection after lung transplantation. Eur Respir J 37: 164-172, 2011.

13. Tamaoki J, Kadota J and Takizawa H: Clinical implications of the immunomodulatory effects of macrolides. Am J Med 117 (Suppl 9A): 5S-11S, 2004

14. Giamarellos-Bourboulis EJ: Macrolides beyond the conventional antimicrobials: A class of potent immunomodulators. Int J Antimicrob Agents 31: 12-20, 2008.

15. Li DQ, Zhou N, Zhang L, Ma P and Pflugfelder SC: Suppressive effects of azithromycin on zymosan-induced production of proinflammatory mediators by human corneal epithelial cells. Invest Ophthalmol Vis Sci 51: 5623-5629, 2010.

16. Council NR: Guide for the care and use of laboratory animals. 8th edition. The National Academies Press, Washington, DC, 2011.

17. Hoffmann N, Lee B, Hentzer M, Rasmussen TB, Song Z, Johansen HK, Givskov M and Høiby N: Azithromycin blocks quorum sensing and alginate polymer formation and increases the sensitivity to serum and stationary-growth-phase killing of Pseudomonas aeruginosa and attenuates chronic P. aeruginosa lung infection in Cftr(-/-) mice. Antimicrob Agents Chemother 51: 3677-3687, 2007.

18. Conte JE Jr, Golden J, Duncan S, McKenna E, Lin E and Zurlinden E: Single-dose intrapulmonary pharmacokinetics of azithromycin, clarithromycin, ciprofloxacin, and cefuroxime in volunteer subjects. Antimicrob Agents Chemother 40: 1617-1622, 1996.

19. Heinzelmann F, Jendrossek V, Lauber K, Nowak K, Eldh T, Boras R, Handrick R, Henkel M, Martin C, Uhlig S, et al: Irradiation-induced pneumonitis mediated by the CD95/CD95-ligand system. J Natl Cancer Inst 98: 1248-1251, 2006

20. Hübner RH, Gitter W, El Mokhtari NE, Mathiak M, Both M, Bolte H,Freitag-Wolf S and Bewig B: Standardized quantification of pulmonary fibrosis in histological samples. Biotechniques 44 : $507-517,2008$

21. Schmittgen TD and Livak KJ: Analyzing real-time PCR data by the comparative C(T) method. Nat Protoc 3: 1101-1108, 2008

22. Fleckenstein K, Gauter-Fleckenstein B, Jackson IL, Rabbani Z, Anscher M and Vujaskovic Z: Using biological markers to predict risk of radiation injury. Semin Radiat Oncol 17: 89-98, 2007.

23. Kergonou JF, Bernard P, Braquet M and Rocquet G: Effect of whole-body gamma irradiation on lipid peroxidation in rat tissues. Biochimie 63: 555-559, 1981.

24. Taysi S, Uslu C, Akcay F and Sutbeyaz MY: Malondialdehyde and nitric oxide levels in the plasma of patients with advanced laryngeal cancer. Surg Today 33: 651-654, 2003.

25. Kang SK, Rabbani ZN, Folz RJ, Golson ML, Huang H, Yu D, Samulski TS, Dewhirst MW, Anscher MS and Vujaskovic Z: Overexpression of extracellular superoxide dismutase protects mice from radiation-induced lung injury. Int J Radiat Oncol Biol Phys 57: 1056-1066, 2003.

26. Aktan B, Taysi S, Gümüştekin K, Uçüncü H, Memişoğullari R, Save $\mathrm{K}$ and Bakan N: Effect of macrolide antibiotics on nitric oxide synthase and xanthine oxidase activities, and malondialdehyde level in erythrocyte of the guinea pigs with experimenta otitis media with effusion. Pol J Pharmacol 55: 1105-1110, 2003.

27. Giaid A, Lehnert SM, Chehayeb B, Chehayeb D, Kaplan I and Shenouda G: Inducible nitric oxide synthase and nitrotyrosine in mice with radiation-induced lung damage. Am J Clin Oncol 26 : e67-e72, 2003

28. Nozaki Y, Hasegawa Y, Takeuchi A, Fan ZH, Isobe KI, Nakashima I and Shimokata K: Nitric oxide as an inflammatory mediator of radiation pneumonitis in rats. Am J Physiol 272 L651-L658, 1997.

29. Mahgoub A, El-Medany A, Mustafa A, Arafah M and Moursi M: Azithromycin and erythromycin ameliorate the extent of colonic damage induced by acetic acid in rats. Toxicol Appl Pharmacol 205: 43-52, 2005.
30. Ward PA and Hunninghake GW: Lung inflammation and fibrosis. Am J Respir Crit Care Med 157: S123-S129, 1998.

31. Hong ZY, Song KH, Yoon JH, Cho J and Story MD: An experimental model-based exploration of cytokines in ablative radiation-induced lung injury in vivo and in vitro. Lung 193 409-419, 2015.

32. Rosiello RA, Merrill WW, Rockwell S, Carter D, Cooper JA Jr, Care S and Amento EP: Radiation pneumonitis. Bronchoalveolar lavage assessment and modulation by a recombinant cytokine. Am Rev Respir Dis 148: 1671-1676, 1993.

33. Kawana A, Shioya S, Katoh H, Tsuji C, Tsuda M and Ohta Y: Expression of intercellular adhesion molecule-1 and lymphocyte function-associated antigen-1 on alveolar macrophages in the acute stage of radiation-induced lung injury in rats. Radiat Res 147: 431-436, 1997.

34. Morgan GW and Breit SN: Radiation and the lung: A reevaluation of the mechanisms mediating pulmonary injury. Int J Radiat Oncol Biol Phys 31: 361-369, 1995.

35. Hong JH, Jung SM, Tsao TC, Wu CJ, Lee CY, Chen FH, Hsu CH, McBride WH and Chiang CS: Bronchoalveolar lavage and interstitial cells have different roles in radiation-induced lung injury. Int J Radiat Biol 79: 159-167, 2003.

36. Chiang CS, Liu WC, Jung SM, Chen FH, Wu CR, McBride WH, Lee CC and Hong JH: Compartmental responses after thoracic irradiation of mice: Strain differences. Int J Radiat Oncol Biol Phys 62: 862-871, 2005.

37. Yamada K, Yanagihara K, Kaku N, Harada Y, Migiyama Y, Nagaoka K, Morinaga Y, Nakamura S, Imamura Y, Miyazaki T, et al: Azithromycin attenuates lung inflammation in a mouse model of ventilator-associated pneumonia by multidrug-resistant Acinetobacter baumannii. Antimicrob Agents Chemother 57: 3883-3888, 2013.

38. Kawashima M, yatsunami J, Fukuno Y, Nagata M, Tominaga M and Hayashi S: Inhibitory effects of 14 -membered ring macrolide antibiotics on bleomycin-induced acute lung injury. Lung 180: 73-89, 2002.

39. Tsoutsou PG and Koukourakis MI: Radiation pneumonitis and fibrosis: Mechanisms underlying its pathogenesis and implications for future research. Int J Radiat Oncol Biol Phys 66: 1281-1293, 2006

40. Wuyts WA, Willems S, Vos R, Vanaudenaerde BM, De Vleeschauwer SI, Rinaldi M, Vanhooren HM, Geudens N, Verleden SE, Demedts MG, et al: Azithromycin reduces pulmonary fibrosis in a bleomycin mouse model. Exp Lung Res 36 602-614, 2010.

41. Bosnar M, Bosnjak B, Cuzic S, Hrvacic B, Marjanovic N, Glojnaric I, Culic O, Parnham MJ and Erakovic Haber V: Azithromycin and clarithromycin inhibit lipopolysaccharide-induced murine pulmonary neutrophilia mainly through effects on macrophage-derived granulocyte-macrophage colony-stimulating factor and interleukin-1beta. J Pharmacol Exp Ther 331: 104-113, 2009.

42. Finkelstein JN, Johnston CJ, Baggs R and Rubin P: Early alterations in extracellular matrix and transforming growth factor beta gene expression in mouse lung indicative of late radiation fibrosis. Int J Radiat Oncol Biol Phys 28: 621-631, 1994.

43. Ianaro A, Ialenti A, Maffia P, Sautebin L, Rombolà L, Carnuccio R, Iuvone $\mathrm{T}$, D'Acquisto $\mathrm{F}$ and $\mathrm{Di}$ Rosa $\mathrm{M}$ : Anti-inflammatory activity of macrolide antibiotics. J Pharmacol Exp Ther 292: 156-163, 2000

44. Martin M, Lefaix J and Delanian S: TGF-betal and radiation fibrosis: A master switch and a specific therapeutic target? Int J Radiat Oncol Biol Phys 47: 277-290, 2000.

45. Vujaskovic Z and Groen HJ: TGF-beta, radiation-induced pulmonary injury and lung cancer. Int J Radiat Biol 76: 511-516, 2000.

46. Xue J, Li X, Lu Y, Gan L, Zhou L, Wang Y, Lan J, Liu S, Sun L, Jia L, et al: Gene-modified mesenchymal stem cells protect against radiation-induced lung injury. Mol Ther 21: 456-465, 2013.

47. Flechsig P, Dadrich M, Bickelhaupt S, Jenne J, Hauser K, Timke C, Peschke P, Hahn EW, Gröne HJ, Yingling J, et al: LY2109761 attenuates radiation-induced pulmonary murine fibrosis via reversal of TGF- $\beta$ and BMP-associated proinflammatory and proangiogenic signals. Clin Cancer Res 18: 3616-3627, 2012

48. Anscher MS, Thrasher B, Rabbani Z, Teicher B and Vujaskovic Z: Antitransforming growth factor-beta antibody 1D11 ameliorates normal tissue damage caused by high-dose radiation. Int J Radiat Oncol Biol Phys 65: 876-881, 2006. 
49. Chen J, He B, Li Y, Wang G and Zhang W: An experimental study on the effect of azithromycin treatment in bleomycin-induced pulmonary fibrosis of rats. Zhonghua Nei Ke Za Zhi 38: 677-680, 1999 (In Chinese)

50. Westermann W, Schöbl R, Rieber EP and Frank KH: Th2 cells as effectors in postirradiation pulmonary damage preceding fibrosis in the rat. Int J Radiat Biol 75: 629-638, 1999.

51. Büttner C, Skupin A and Rieber EP: Transcriptional activation of the type I collagen genes COL1A1 and COL1A2 in fibroblasts by interleukin-4: Analysis of the functional collagen promoter sequences. J Cell Physiol 198: 248-258, 2004.
52. Vos R, Vanaudenaerde BM, Verleden SE, Ruttens D, Vaneylen A, Van Raemdonck DE, Dupont LJ and Verleden GM: Anti-inflammatory and immunomodulatory properties of azithromycin involved in treatment and prevention of chronic lung allograft rejection. Transplantation 94: 101-109, 2012.

53. Radhakrishnan SV, Palaniyandi S, Mueller G, Miklos S, Hager M, Spacenko E, Karlsson FJ, Huber E, Kittan NA and Hildebrandt GC: Preventive azithromycin treatment reduces noninfectious lung injury and acute graft-versus-host disease in a murine model of allogeneic hematopoietic cell transplantation. Biol Blood Marrow Transplant 21: 30-38, 2015. 